\title{
SLE IN PREGNANCY
}

ISLAM F $^{2}$, BEGUM N $^{2}$

Systemic lupus erythematosus (SLE) is an autoantibody-mediated systemic autoimmune disease, predominantly affecting young females in there childbearing ages. lupus pregnancy is labeled as 'high-risk' pregnancy. Disease flare of SLE can occur during pregnancy. Pregnancy related complications are common with SLE. Fetal outcome is also poor. There is a increased risk of spontaneous miscarriage, intra-uterine growth restriction, fetal death, neonatal lupus syndrome, preeclampsia and pre-term delivery. Over the years, the pregnancy outcomes have significantly improved. In recent years the rate of pregnancy loss has decreased from $43 \%$ to $17 \% .{ }^{1}$ SLE is not itself a contra-indication to pregnancy, with the exception of organ-system complications such as pulmonary hypertension and renal failure, advanced heart failure, previous severe preeclampsia or HELLP syndrome despite treatment. Active disease and lupus nephritis increase the risk of fetal loss and other adverse outcomes ${ }^{2}$. Proteinuria, hypertension, thrombocytopenia, and presence of anti-phospholipid antibodies are other negative predictors for fetal survival 3 . Fertility is normal as autoimmune ovarian failure is rare. menstrual disorders or ovarian failure may occur in end-stage renal disease, drugs used to treat SLE (e.g. cyclophosphamide).

Flares may occur during all three trimesters with equal frequency, and often in the immediate postpartum period. It is not possible to predict when, or if, an individual patient will flare. Flare is more likely if disease has been active within 6 months of conception. The main predictor of a flare during pregnancy and its degree is the number and the severity of flares before conception. Prophylactic steroids does not lower the frequency of flares. The fatal complications of SLE flare are renal involvement and CNS manifestation.

Many physiological changes of pregnancy may overlap with features of active disease, making differentiation difficult. Some common laboratory tests also become less reliable: mild anemia and thrombocytopenia are common, erythrocyte sedimentation rate is raised, and up to $300 \mathrm{mg} /$ day proteinuria can occur during normal pregnancy. Complement levels rise by 10-50\% during normal pregnancy and may appear to remain in the 'normal' range, despite disease activity. Thus, the trend of complement levels becomes more important than absolute values. Low sand declining levels of complement during pregnancy found to be associated with poor pregnancy outcomes ${ }^{4}$. Anti-dsDNA antibodies may be helpful in evaluation of disease activity ${ }^{4}$.

Active SLE at the time of conception is the strongest predictor of adverse pregnancy outcomes $^{5}$. So, in women with SLE pregnancies should be planned during periods of disease control. Prior to conception a management strategy should be agreed in full consultation with the patient. Thyroid function should be assessed as ${ }^{1}$ hypothyroidism in SLE is associated with poorer outcomes ${ }^{6}$. They should be counseled about the risk of disease flares, higher rates of pregnancy complications, poor obstetric outcomes, the risk of neonatal lupus syndromes and the need for optimal disease control with safe medications during pregnancy.

1. Professor Ferdousi Islam, Head of Dept. of Obs \& Gynae, Dhaka Medical College.

2. Dr. Nazneen Begum, Assistant Professor, Dept. of Obs \& Gynae, Dhaka Medical College. 
Patient should be evaluated in a comprehensive and up-to-date manner for

1. Major organ function- If severe dysfunction is present, pregnancy is not advised. Special emphasis is given on the kidneys and thrombocytopenia.

2. Assessment of disease activity - if active disease pregnancy is deferred, if stableadvised for pregnancy.

3. Autoantibody profile- for risk evaluation aPL $\&$ anti Ro antibody to be done.

4. Review medication and adjust to achieve optimal control on safe drugs before conception.

Women with SLE should be followed up by an obstetrician knowledgeable in high-risk pregnancies. Frequency of clinic visits depends upon need, but the recommendation is a minimum of monthly visits until 28 weeks, fortnightly visits to 36 weeks and then weekly visits. Baseline tests should include: complement studies (C3, C4, CH5O), antidsDNA, anticardiolipin antibodies, lupus anticoagulant (Russell viper venom time), antiSSA and SSB (Ro and La), microscopic urinalysis, a 24-hour urine collection to determine total protein, total calcium, creatinine clearance, and protein-tocreatinine ratio.

Monthly measurement of: complements $\mathrm{C} 3$ and C4, ds-DNA antibodies, aPL, biochemical profile, full blood count, urinalysis. 24-hr urine collection for creatinine clearance and proteinuria quantification in women with preexisting renal disease. Regular sonogram is needed for measurement of amniotic fluid volume and fetal growth. Doppler assessment of uterine artery blood flow at 20-24 weeks is useful in predicting pre-eclampsia and intrauterine growth restriction. Biophysical profile (BPP) scoring to be started from 28 weeks. If anti-Ro or anti-La antibodies are positive then serial foetal echocardiograms between 16-24 weeks of gestation is offered.11 Women who show evidence of increased serologic activity but remain asymptomatic should be monitored more closely. There is no need to initiate therapy for serologic findings alone, with the possible exception of the development of aPL. During pregnancy, C3 and C4 may rise above normal levels, and thus a flare with complement activation may occur despite apparently normal levels of C3 and C4.

To prevent flare mother should avoid sun exposure. She should take adequate amount of vitamins and minerals and folic acid. Hydroxychloroquine should be continued in all SLE pregnancies, discontinuation led to an increase in disease flares. It reduces risk of disease flares, CHB and NLS (Neonatal Lupus Syndrome ). Low-dose aspirin as an antiplatelet agent is safe during pregnancy but data on other antiplatelet agents are limited .NSAID should be used with caution during the first and second trimester \& discontinued during last trimester. Use of NSAID sin first trimester may be associated with higher risk of congenital malformations, fetal renal impairment and in third trimester premature closure of ductus arteriosus.

Steroid exposure should be minimum during the pregnancy. High doses are associated with an increased risk of fetal cleft palate, IUGR, diabetes, hypertension, pre-eclampsia and premature rupture of membranes. But during disease flares, high doses and/or intravenous pulse methylprednisolone to be used for short period. Patients on long term steroid therapy should also receive stress doses at the time of delivery. When premature delivery is anticipated single course of fluorinated compounds, such as dexamethasone and betamethasone should be given for fetal lung maturity. Repeated use is associated with impaired neuro-psychological development of the child in later life. If by fetal doppler incomplete heart block is detected, to decrease inflammation in the fetal heart and prevent progression to complete heart block corticosteroids that cross the placenta (dexamethasone or betamethasone) to be administered to the mother ${ }^{7}$. Plasmapheresis and IVIG are also tried for prevention of CHB. (Successful prevention)

Azathioprine is an immunosuppressive agent that is safe during pregnancy. The dose should be not more than $2 \mathrm{mg} / \mathrm{kg} /$ day, to avoid risk of 
fetal cytopenias and immune suppression. It is associated with late developmental delays in offsprings. Calcineurin inhibitors, tacrolimus and cyclosporine are other immunosuppressive drugs with no reported increase in fetal risk. Cyclophosphamide, methotrexate, and mycophenolate, are contraindicated during pregnancy and should be discontinued at least 3 months before conception. Data on the biologics, such as rituximab or belimumab, during pregnancy are very limited, and they should be discontinued before conception.

Before conception or in early pregnancy antihypertensive medications should be switched to those that are safe in pregnancy (methyldopa, labetalol and nifedipine). Angiotensin-converting-enzyme inhibitors and Angiotensin-2-receptor antagonists are associated with renal dysfunction in the fetus and therefore should be avoided. Diuretics decrease intravascular volume, hence should be avoided.If loop diuretics are needed to maintain renal function, they should be continued. Frusemide is the loop diuretic with the best track record for treatment of chronic renal failure in pregnancy. It poses a low risk to the fetus.

If antiphospholipid syndrome is present, there is greatly increased risk of thrombosis and foetal loss.Heparin does not cross the placenta and is the anticoagulant of choice during pregnancy. Warfarin should be avoided during pregnancy, especially during the first trimester, due to the risk of warfarin embryopathy syndrome.Calcium supplementation should be given routinely to all pregnant women with SLE, especially those receiving corticosteroids and heparin. Insufficient vitamin D levels during pregnancy are associated with higher pregnancy morbidity including gestational diabetes, pre-eclampsia, and small for gestational age infants. ${ }^{8}$ Bisphosphonates should be discontinued 6-12 months prior to pregnancy.

If a mother get pregnant during the treatment of SLE with MTX or cylophosphamide, diagnostic tests should be done for early detection of teratogenicity, like-Chorionic villus sampling,
Amniocentesis, Cordocentesis 3-D ultrasound, Fetal MRI; which one is suitable for that gestational period. If fetal condition is untreatable or associated with significant handicap then termination of pregnancy is advised. Termination is also advised if pregnancy endangers her life. Delivery should be done in such hospital where neonatal ICU is available. Indication of caesarean section same as normal pregnancy, include maternal reasons (avascular necrosis of the hips with inadequate hip abduction) or foetal reasons (foetal distress, abnormal nonstress test, cephalo-pelvic disproportion and transverse presentation etc.).

After delivery heart rate of the baby should be counted and also there should be a search for any cutaneous lesion.

Treatment of established congenital heart block (CHB) is difficult23. Therefore, it is better to prevent during pregnancy. For cutaneous lesion with topical steroids can be applied. Most of drugs are excreted in breast milk in variable amounts. maternal intake of less than or up to $30 \mathrm{mg} /$ day of prednisolone, warfarin, cyclosporine in standard doses and weekly chloroquine for malaria prophylaxis are considered safe for breastfed babies. If the dose of prednisolone is greater than $30 \mathrm{mg} /$ day, feeding should be avoided for 4 hours after ingestion of the morning dose of steroid. By this time the blood levels are low and very limited amounts are secreted into the milk. If mother is on cyclophosphamide, azathioprine, hydroxychloroquine for SLE breastfeeding is contraindicated.

During the postpartum period, the mother should be carefully followed up for any sign of infection and disease exacerbation; both require aggressive treatment. In Antiphospholipid antibody Syndrome, warfarin is restarted

after bleeding stops. Barrier methods are the safest method for contraception. However, low dose estrogen or progesterone only pills are relatively safe. High dose estrogen containing pill should be avoided. OCP should be avoided in antiphospholipid syndrome, other 
thromboembolic diseases, highly active disease, migraine, Raynaud's phenomenon. Intrauterine devices use causes infections like endometritis, PID etc.

SLE is a multisystem disease. So a multidiscciplinary approach, with close medical, obstetric and neonatal monitoring, is essential for optimal outcomes.

J Dhaka Med Coll. 2015; 24(2) : 82-85

\section{References:}

1. Clark CA, Spitzer KA, Laskin CA. Decrease in pregnancy loss rates in patients with systemic lupus erythematosus over a 40 -year period. J Rheumatol. 2005;32(9):1709-1712.

2. Wagner SJ, Craici I, Reed D, Norby S, Bailey K, Wiste HJ, Wood CM, Moder KG, Liang KP, Liang $\mathrm{KV}$, et al. Maternal and foetal outcomes in pregnant patients with active lupus nephritis. Lupus. 2009; 18(4):342-347.

3. Lockshin MD, Kim M, Laskin CA, Guerra M, Branch DW, Merrill J, Petri M, Porter F, Sammaritano L, Stephenson MD, et al. Lupus anticoagulant, but not anticardiolipin antibody, predicts adverse pregnancy outcome in patients with antiphospholipid antibodies. Arthritis Rheum. 2012 doi: 10.1002/art.34402.

4. Clowse ME, Magder LS, Petri M. The clinical utility of measuring complement and anti-dsDNA antibodies during pregnancy in patients with systemic lupus erythematosus. J Rheumatol. 2011;38(6):1012-1016.

5. Lateef A, Petri M. Management of pregnancy in systemic lupus erythematosus. Nat Rev Rheumatol.2012;8(12):710-718.

6. Stagnaro-Green A, Akhter E, Yim C, Davies TF, Magder L, Petri M. Thyroid disease in pregnant women with systemic lupus erythematosus: increased preterm delivery. Lupus. 2011;20(7):690-699.

7. Motha MBC, Wijesinghe PS. Systemic lupus erythematosus and pregnancy- a challenge to the clinician. Ceylon J Med 2009; 54(4): 107-9

8. Aghajafari F, Nagulesapillai T, Ronksley PE, Tough SC, O'Beirne M, Rabi DM. Association between maternal serum 25-hydroxyvitamin D level and pregnancy and neonatal outcomes: systematic review and meta-analysis of observational studies. BMJ. 2013;346:f1169. 